\title{
Subtype Classification of Hepatocellular Adenoma
}

\author{
Paulette Bioulac-Sage ${ }^{a, b}$ Charles Balabaud ${ }^{b, c}$ Jessica Zucman-Rossid, e \\ aService d'Anatomie Pathologique, Hôpital Pellegrin, CHU Bordeaux, 'b Inserm, U889, Université Bordeaux 2, and \\ 'Service d'hépatologie, Hôpital St André, CHU Bordeaux, dUniversité Paris Descartes, Faculté de médecine, AP-HP,

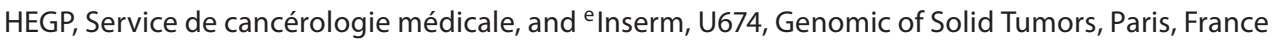

\section{Key Words}

Hepatocellular adenomas $\cdot$ HCA subgroups - Genotype/ phenotype classification $\cdot H N F 1 A$ gene $\cdot \beta$-Catenin gene $\cdot$ gp130 gene

\begin{abstract}
Hepatocellular adenomas (HCA) are rare benign tumours occurring mainly in women under oral contraceptives. HCA bleed frequently and transform rarely into hepatocellular carcinoma. Identification of genes recurrently mutated in HCA and good genotype/phenotype correlations provided the basis of a pathomolecular classification of different HCA subgroups, characterized using immunohistochemical markers. HNF1A-mutated HCA: Biallelic-inactivating mutations of HNF1A gene are identified in $35-40 \%$ of HCA. HNF1 $\alpha$-inactivated HCA display characteristic pathological features, including marked steatosis. The expression of FABP1 (which is a HNF1A target gene) is downregulated and the absence of L-FABP expression diagnosed this subgroup. $\beta$-Catenin-mutated HCA: $\beta$-catenin mutations leading to activation of the $\mathrm{Wnt} / \beta$-catenin pathway represented $10-15 \%$ of HCA. They are characterized by overexpression of glutamine synthetase and aberrant nuclear $\beta$-catenin staining. These $\beta$-catenin-activated HCA are at greater risk of malignant transformation; they are difficult to differentiate from well-differentiated HCC. Inflammatory HCA (50\%): These are
\end{abstract}

defined by the presence of inflammatory infiltrates, sinusoidal dilatation and thick-walled arteries. Small in-frame deletions that target the binding site of gp130 for IL- 6 have been reported in $60 \%$ of inflammatory HCA. There is an overexpression of the inflammatory proteins serum amyloid $A$ and C-reactive protein in tumour hepatocytes both at mRNA and protein levels. Inflammatory HCA occurred more frequently in patients with high body mass index; they can be also mutated for $\beta$-catenin and therefore are probably at risk of HCC. Unclassified HCA: Less than 10\% of HCA do not express any of the above-mentioned phenotypic markers. Taking into account noticeable differences between the HCA subgroups, in terms of clinical and prognostic features, phenotyping may become an important tool for HCA management strategy.

Copyright $\odot 2010$ S. Karger AG, Basel

Hepatocellular adenoma (HCA) is a rare benign tumour, occurring mainly in women taking oral contraceptives. These tumours are rare in children, men and the elderly. Their incidence is around 3-4/100,000 in Europe and North America [1]. In addition to oral contraceptives, other risk factors have been identified including androgens, glycogenosis, tyrosinaemia, familial polyposis coli. HCA are composed of hepatocytes which are arranged in liver cell plates that are only mildly thickened

\section{KARGER}

Fax +41613061234

E-Mail karger@karger.ch

www.karger.com
(C) 2010 S. Karger AG, Basel

0253-4886/10/0271-0039\$26.00/0

Accessible online at:

www.karger.com/dsu
Paulette Bioulac-Sage

Service d'anatomie pathologique, Hôpital Pellegrin, CHU Bordeaux

Place Amélie Raba Leon, FR-33076 Bordeaux Cedex (France)

Tel. +335567956 02, Fax +33556796088

E-Mail paulette.bioulac-sage@chu-bordeaux.fr 
or irregular. The tumour parenchyma is supplied by numerous arteries which are unaccompanied by bile ducts. Tumour hepatocytes are of normal size; the cytoplasm may be either normal, clear (glycogen-rich) or fatty. Nuclear atypia and mitoses are distinctly unusual. In contrast, changes including sinusoidal dilatation, peliosis, infarcts, and haemorrhage are frequent, and may result in oedematous or fibrotic regions. There are two types of haemorrhage which are mostly observed in HCA $>4-5$ $\mathrm{cm}$ : (i) haemorrhage inside the HCA nodule, usually admixed with necrotic changes, and (ii) spontaneous haemorrhagic rupture which can cause subcapsular haematoma and possible haemoperitoneum. Hepatocellular carcinoma may arise in HCA, but this is of rare occurrence. The diagnosis of HCA can usually be made on routine HE staining, but pathological variations are frequently seen and immunohistochemistry is essential for classification into different subtypes [2], whose recognition is important for the follow-up and management of the patient [3].

\section{Genotype/Phenotype Classification of Hepatocellular Adenoma}

HCA represents a heterogenous entity, recently subclassified into several groups according to their genotype and phenotype. This classification was proposed following a large multicentric French study of molecular markers of HCA and their correlation with microscopic pathologic features [4]. In our institution, more than $130 \mathrm{HCA}$ resected between 1984 and 2008 have been reviewed and classified according to genotype/phenotype and immunohistochemical characteristics [3]. At the present time, 5-10 HCA are still being resected annually.

\section{Genetics}

HCA are monoclonal tumours in which several recurrent mutations have been identified [4-7].

\section{HNF1A (Also Called TCF1) Gene}

The HNF1A gene encodes the hepatocyte nuclear factor $1(\mathrm{HNF} 1) \alpha$. Biallelic-inactivating mutations of this gene have identified $H N F 1 A$ as a tumour suppressor gene involved in liver tumorigenesis [5]. HNF1A germline mutations confer a predisposition to both diabetes (MODY3) and liver adenomatosis [8]. Recently, CYP1B1 heterozygous germline-inactivating mutations were identified in
$15 \%$ of women that have HNF1A-mutated HCA (HHCA), suggesting that germline CYP1B1 mutations may also confer predisposition to the development of sporadic H-HCA [9].

\section{$\beta$-Catenin Gene}

$\beta$-Catenin-activating mutations have been identified in a number of HCA cases $(\beta$-HCA) $[4,6]$. $\beta$-Catenin mutation leads to the activation of the Wnt $/ \beta$-catenin pathway that plays a key role in liver development and physiology, such as lineage specification, differentiation, stem cell renewal, epithelial-mesenchymal transition, zonation, proliferation, cell adhesion and liver regeneration. Mutations of $\beta$-catenin are also found in $20-34 \%$ of hepatocellular carcinomas.

\section{gp130 Gene}

It has recently been shown [7] that some HCA are associated with gain-of-function mutations caused by inframe somatic deletions in the IL6ST gene. This gene encodes for the signalling co-receptor gp130. Mutant gp130 constitutively activates STAT3 signalling in the absence of IL- 6 binding. gp130 and $\beta$-catenin mutations may coexist in a same HCA.

\section{HCA Subgroups According to Genotype/Phenotype Classification}

Group 1: Hepatocellular Adenomas with Mutations in the HNF1A Gene: H-HCA

HNF1A mutations are observed in approximately 35$40 \%$ of HCA. Biallelic-inactivating HNF1A mutations have been identified in tumour tissues: in most cases, both mutations are of somatic origin, whereas in less than $10 \%$ of cases, one mutation is of germline origin while the other is somatic. Patients with germline HNF1A mutations are younger than those with somatic mutations and they frequently have a family history of liver adenomatosis [8]; they may or may not have clinical diabetes, which is usually of the MODY3 type.

Correlations with pathological and clinical data have shown that H-HCA mostly correspond to a histologically homogeneous group of tumours, characterized by marked steatosis, no cytological abnormalities, no inflammatory infiltrates (fig. 1). Furthermore, $F A B P 1$, which is a gene positively regulated by $H N F 1 A$, and expressed in normal liver tissue, displays downregulated expression levels in H-HCA. Correlatively, using immunohistochemistry, we observed a lack of LFABP expres- 


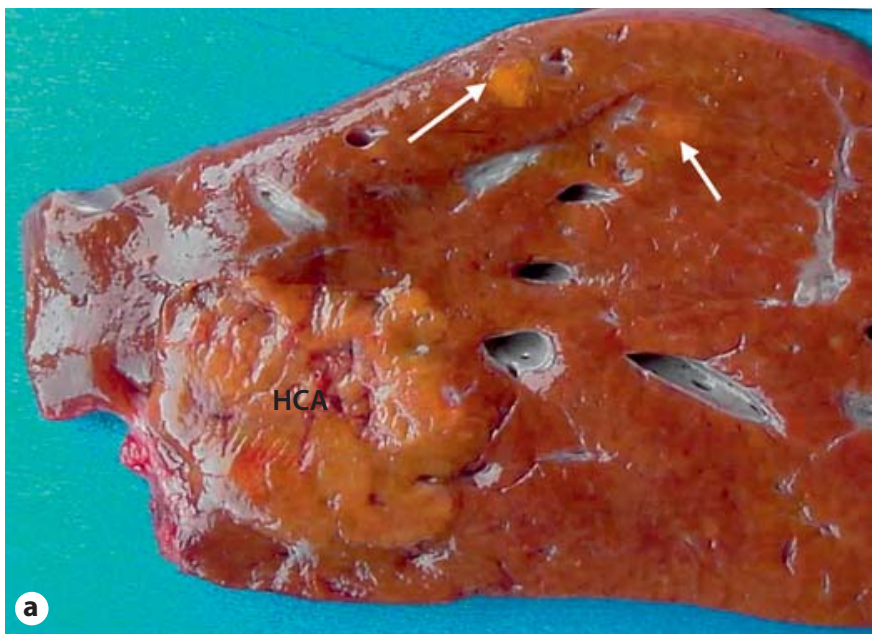

Fig. 1. HNF1 $\alpha$-inactivated HCA in a 49-year-old woman. a Resected fresh specimen: $40 \mathrm{~mm}$, yellowish tumour of segment III, with lobulated contours; pale microadenomas are visible in the surrounding liver (arrows). b HCA is highly steatotic, non-encapsulated, with a clear demarcation from the non-tumoural liver (NTL). c LFABP immunostaining: lack of LFABP in HCA, contrasting with normal expression in NTL.

sion in the tumour which contrasted with normal expression in the surrounding non-tumoural liver [2]. The sharp contrast between tumour and adjacent liver in terms of steatosis and LFABP expression enables delineation of tumour borders which are often irregular and lobulated, with often small HCA foci present in the immediate surrounding parenchyma. When liver adenomatosis is observed, multiple adenomas of varying size are present, associated with a myriad of steatotic micronodules. The latter should not be misinterpreted as hepatic steatosis. All these micronodules can be correctly identified by lack of LFABP staining. Somatic H-HCA occurs almost exclusively in women.

Group 2: Hepatocellular Adenomas with Mutations of the $\beta$-Catenin Gene: $\beta$-HCA

Approximately $10-15 \%$ of HCA demonstrate an activating $\beta$-catenin gene mutation, which can be demon-
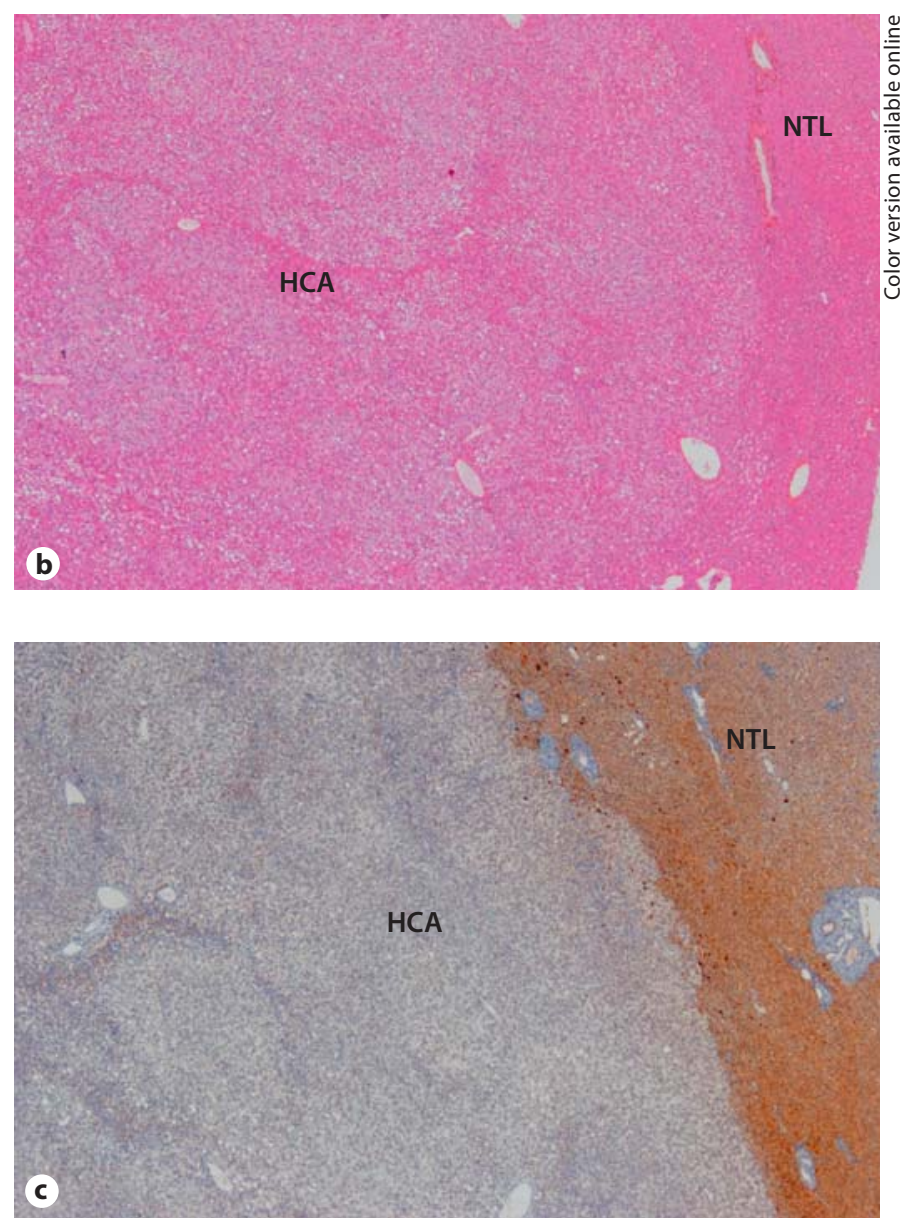

strated by immunohistochemistry. The usual pattern results in an aberrant nuclear and cytoplasmic staining, distributed in a random and heterogenous pattern (fig. 2). Furthermore, Glul, a $\beta$-catenin target gene, coding for glutamine synthetase, also displays upregulated expression. Therefore, these $\beta$-HCA show also strong and diffuse glutamine synthetase staining [2].

Correlations with corresponding pathological and clinical data have shown that tumours with $\beta$-catenin mutations are usually characterized by the presence of cytological abnormalities and acinar pattern, whereas they are not steatotic [4]. Due to the cytological and architectural abnormalities, these tumours are often extremely difficult to distinguish from well-differentiated HCC. Moreover, they are also more frequently associated with the development of unequivocal HCC than other HCA subtypes [2-4, 10]. Furthermore, this subgroup of $\beta$-HCA is overrepresented in male patients and specific 

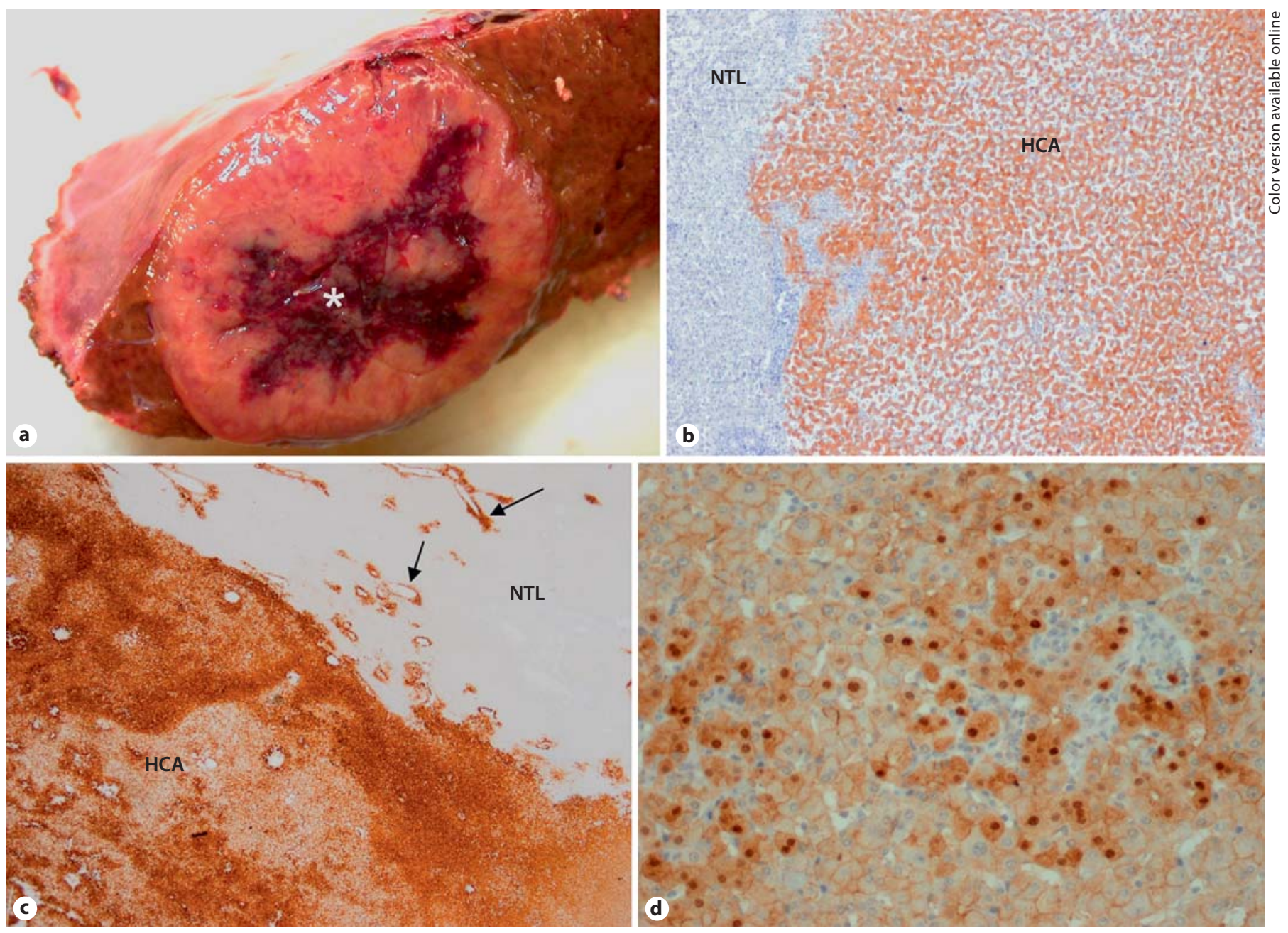

Fig. 2. Inflammatory and $\beta$-catenin-activated HCA in a 30-yearold woman. a Resected fresh specimen: $35-\mathrm{mm}$ tumour of segment VII, presenting a large congestive area $\left(^{*}\right)$. b CRP immunostaining: CRP is overexpressed by hepatocytes of the HCA, with a clear demarcation from the non-tumoural liver (NTL). c GS

immunostaining: strong and diffuse expression of GS by HCA, contrasting with the NTL where GS is expressed only by a few centrolobular hepatocytes (arrow). d $\beta$-Catenin immunostaining: focally aberrant nuclear and cytoplasmic expression by some adenomatous hepatocytes.

risk factors are often found, such as male hormone administration, glycogenosis, and familial polyposis. However, death due to HCC was more often linked to women with this type of $\beta$-HCA in our own series [3].

\section{Group 3: Inflammatory Hepatocellular Adenomas:}

IHCA

IHCA account for more than $50 \%$ of all HCA, and are associated with high body mass index and alcohol consumption $[3,11,12]$. These patients may have signs and symptoms of an inflammatory syndrome, including elevated serum CRP levels [13]. These tumours are characterized morphologically by the presence of inflammatory

infiltrates, marked sinusoidal dilatation or congestion, numerous thick-walled arteries, and a more or less obvious ductular reaction, lying inside a small amount of connective tissue (fig. 3). This subgroup of HCA includes most of the previously termed 'telangiectatic focal nodular hyperplasia' [11] or telangiectatic adenoma [12]. In this group, overexpression of molecules of the acute-phase inflammatory response, such as serum amyloid A (SAA) and C-reactive protein (CRP), at both the mRNA and protein levels is observed [2]. Immunohistochemical detection of these proteins (SAA and CRP) in tumoural hepatocytes is a very good argument for the diagnosis of IHCA [2]. 


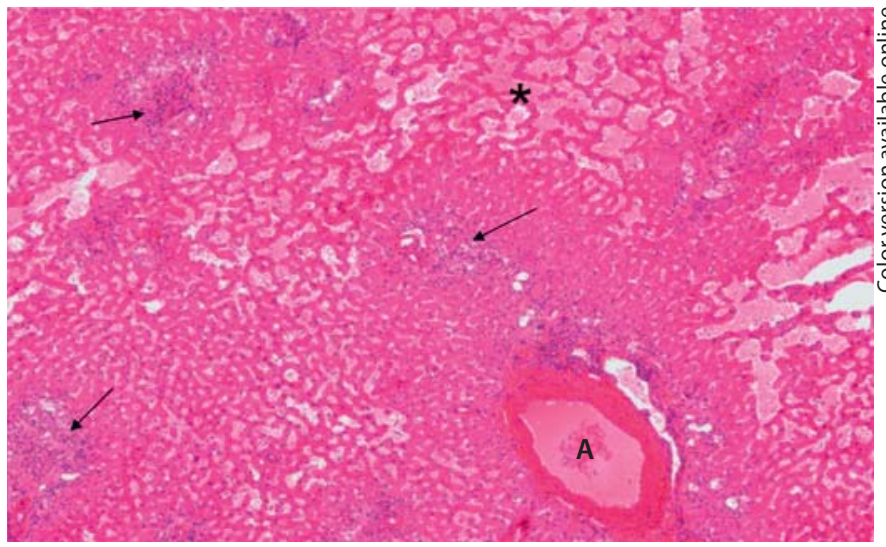

Fig. 3. Inflammatory HCA. Typical microscopic features: sinusoidal dilatations $\left(^{*}\right)$, inflammatory infiltrates (arrows), thick-walled arteries (A).

The extent of congestion, peliosis and haemorrhage varies from case to case. Steatosis may be present in IHCA, but is usually not as extensive as in the H-HCA. When present, steatosis is usually distributed irregularly within the IHCA, and when there are multiple tumours, the amount of steatosis varies among the tumours in an individual patient. Despite their morphological aspect, these IHCA express both inflammatory proteins SAA and CRP with a sharp demarcation from the surrounding non-tumoural liver [2]. Positive hepatocytes are not necessarily located adjacent to inflammatory cells. Approximately $10 \%$ of IHCA have a mutation in the $\beta$-catenin gene. The risk of HCC does also exist in these inflammatory $/ \beta-H C A$, but in a proportion that is presently unknown.

\section{Group 4: Hepatocellular Adenoma without Markers}

Tumours in this group, representing less than $5-10 \%$ of cases, neither show HNF1A nor $\beta$-catenin gene mutations and do not express inflammatory proteins. An additional group includes HCA that cannot be classified due to near-total necrosis or haemorrhage.

\section{Differential Diagnosis}

Differential diagnosis may be challenging, mainly in a liver biopsy; in particular the distinction of $\mathrm{FNH}$ versus HCA, HCA versus well-differentiated HCC, steatotic FNH or steatotic HCA versus focal fatty changes and even FNH or HCA versus normal liver. Immunohisto- chemistry as detailed above is very useful in these situations provided that tumoural and non-tumoural liver tissue is available for comparison.

Furthermore, the two lesions FNH and HCA may coexist [14], especially when there are multiple nodules, e.g. adenomatosis and FNH, or multiple FNH and HCA. Errors are best avoided by assaying a panel of immunohistochemical markers $[2,15]$ and interpreting these in the appropriate clinical, biological and radiological context with the benefit of expertise from a team with a special interest in these hepatocellular nodules. It is always careful to freeze tissue for molecular characterization. In the end, it is likely that a few nodules may remain unclassified (or even misclassified).

\section{Investigation of HCA Subtypes}

The main complications of HCA are bleeding which occurs in $20-25 \%$ of cases and HCC in $5-9 \%$ of cases [3-10]. Surgery remains the main treatment to avoid these complications [16-18]. Currently the main characteristic to guide treatment remains the size of the nodule with excision for nodules $\geq 5 \mathrm{~cm}$ and surveillance for nodules $<5 \mathrm{~cm}[3,10]$. It is likely that the phenotypic classification will refine this clinical practice.

\section{HCC}

There is a small but definite incidence of malignant transformation of HCA $[3,4,10]$. Although this is sometimes debated, there is enough evidence today to be certain that HCA can transform into HCC. This includes the co-existence of both HCA and HCC within the same tumour and the occurrence of an overt HCC several years after partial resection of a 'true' HCA. However, the exact pathways of malignant transformation and the exact point at which the switch to malignant transformation occurs remain unknown. The risk of malignancy is particularly higher for $\beta-\mathrm{HCA}$, which are most frequently associated with glycogenosis type 1 , androgenic hormone intake, and familial adenomatous polyposis. This subtype of HCA constitutes a significant number of tumours in men. Since $\beta$-HCA often show cytological atypia and pseudoglandular pattern, it is often very difficult and sometimes almost impossible to differentiate these from a well-differentiated HCC. Although glypican-3 is a promising marker for the identification of HCC, its utility is limited by the fact that it may not be detected in approximately one third of HCCs which develop in a normal liver. In inflammatory HCA, we sus- 
Fig. 4. Imaging and needle biopsy for diagnosis of HCA subtypes. * Taking into account context, size $(<$ or $>5 \mathrm{~cm})$, location, sex, aetiology, age. ${ }^{+}$Specialized liver centres (radiology, pathology). ${ }^{*}$ The differential diagnosis between HCA and welldifferentiated HCA remains difficult.

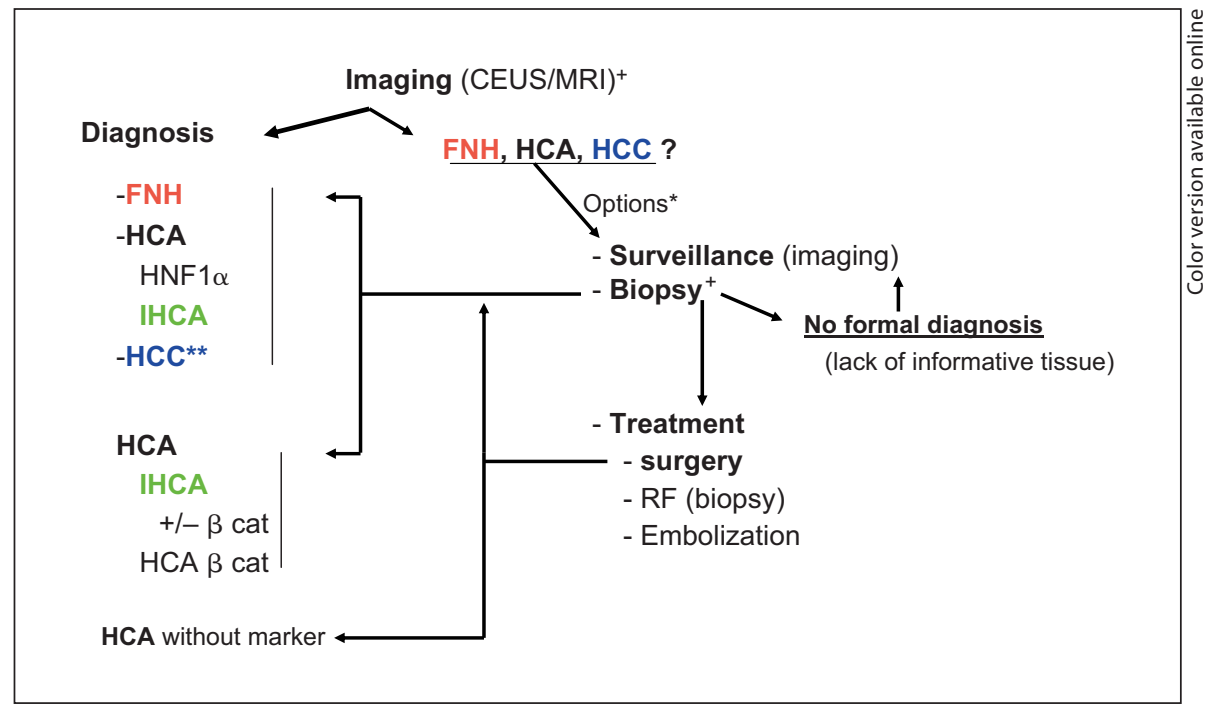

pect that $\beta$-catenin mutation is a secondary event that occurs at a late time. We observed that men were distributed almost exclusively in the IHCA subgroup with or without activation of the $\beta$-catenin pathway. These tumours occurring in men were always solitary with no microadenomas in the surrounding liver. The associated factors include alcohol, tobacco, elevated BMI and are those also encountered in HCC on non-cirrhotic liver [3]. The risk of HCC appears high enough so that destruction of even small nodule $(<5 \mathrm{~cm})$ may be reasonable in men.

\section{Haemorrhage}

Recent data seem to indicate that haemorrhage is more frequent in HCA with sinusoidal dilatation and congestion [10]

\section{Adenomatosis}

LFABP-negative HCA and IHCA are the only two subgroups with a wide range of nodules per case, from solitary to multiple $(<$ and $>5)$. There is no additional risk of bleeding or HCC transformation whether the nodule is solitary or multiple. The number of detectable HCA is less that the true number of HCA since microadenomas are not detected by conventional imaging. The term adenomatosis - if kept - which should mean many HCA, is not a specific type of HCA as thought previously $[19,20]$. From a clinical perspective, size, genotype, and underlying disease are far more important than the number of lesions.
It has been shown that in patients presenting multiple HCA, the tumours are of the same subtype in terms of expression of LFABP or inflammatory proteins, whatever the size and number of nodules [21,22]. On the contrary, $\beta$-catenin expression is not found in all nodules, suggesting that $\beta$-catenin mutation might be a secondary event in tumorigenesis. It also underscores the fact that multiple nodules, and probably the largest, should be sampled to detect $\beta$-catenin mutation which indicates a higher risk of malignant transformation.

\section{Follow-Up}

It is expected that the phenotype classification will have values for the follow-up of nodules $<5 \mathrm{~cm}$. We confirmed that non-resected HCA (usually of small size) remain for their great majority stable, tend to disappear in a small percentage of cases and in general do not grow. The observation that IHCA may disappear more rapidly is interesting and needs to be confirmed. Follow-up of patients with LFABP-negative HCA as well as IHCA with complete resection can reasonably be stopped few years after surgery; for those with incomplete resection and with no significant change in HCA size during the first years, the follow-up can probably be performed at longer intervals [3].

\section{Respective Role of Imaging and Needle Biopsy for the} Diagnosis of HCA Subtypes

We have shown that H-HCA and inflammatory HCA were associated with specific MRI patterns related to diffuse fat repartition and sinusoidal dilatation, respectively 
[23]. When imaging techniques fail to identify liver nodules, it is time for a liver biopsy (fig. 4). Liver biopsy is of help to identify HCA subtypes. Difficulties may occur mainly because the tissue sample is not representative. The ultimate result will be given using the surgical specimen.

\section{Conclusion}

The major importance of the genotype/phenotype classification needs to be confirmed by additional studies particularly from teams from other countries where the use of oral contraceptives is less common.

\section{References}

1 Barthelmes L, Tait IS: Liver cell adenoma and liver cell adenomatosis. HPB (Oxford) 2005; 7:186-196.

-2 Bioulac-Sage P, Rebouissou S, Thomas C, Blanc JF, Saric J, Sa Cunha A, et al: Hepatocellular adenoma subtype classification using molecular markers and immunohistochemistry. Hepatology 2007;46:740-748.

-3 Bioulac-Sage P, Laumonier H, Couchy G, Le Bail B, Sa Cunha A, Rullier A, Laurent C, Blanc JF, Cubel G, Trillaud H, Zucman-Rossi J, Balabaud C, Saric J: Hepatocellular adenoma management and phenotypic classification: the Bordeaux experience. Hepatology 2009;50:481-489.

$\checkmark 4$ Zucman-Rossi J, Jeannot E, Nhieu JT, Scoazec JY, Guettier C, Rebouissou S, et al: Genotype-phenotype correlation in hepatocellular adenoma: new classification and relationship with HCC. Hepatology 2006;43: 515-524.

5 Bluteau O, Jeannot E, Bioulac-Sage P, Marqués JM, Blanc JF, Bui H, Beaudoin JC, Franco D, Balabaud C, Laurent-Puig P, ZucmanRossi J: Bi-allelic inactivation of TCF1 in hepatic adenomas. Nat Genet 2002;32:312315.

6 Rebouissou S, Bioulac-Sage P, Zucman-Rossi J: Molecular pathogenesis of focal nodular hyperplasia and hepatocellular adenoma. J Hepatol 2008;48:163-170.

-7 Rebouissou S, Amessou M, Couchy G, Pous$\sin \mathrm{K}$, Imbeaud S, Pilati C, et al: Frequent inframe somatic deletions activate gp130 in inflammatory hepatocellular tumours. Nature 2009;57:200-204

$\checkmark 8$ Bacq Y, Jacquemin E, Balabaud C, Jeannot E, Scotto B, Branchereau S, et al: Familial liver adenomatosis associated with hepatocyte nuclear factor- $1 \alpha$ inactivation. Gastroenterology 2003;125:1470-1475.
9 Jeannot E, Poussin K, Chiche L, Bacq Y, Sturm N, Scoazec JY, Buffet C, Van Nhieu JT, Bellanné-Chantelot C, de Toma C, LaurentPuig P, Bioulac-Sage P, Zucman-Rossi J: Association of CYP1B1 germ line mutations with hepatocyte nuclear factor-1 $\alpha$-mutated hepatocellular adenoma. Cancer Res 2007; 67:2611-2616

10 Dokmak S, Paradis V, Vilgrain V, Sauvanet A, Farges O, Valla D, Bedossa P, Belghiti J: A single-center surgical experience of $122 \mathrm{pa}$ tients with single and multiple hepatocellular adenomas. Gastroenterology 2009;137 1698-1705.

-11 Bioulac-Sage P, Rebouissou S, Sa Cunha A, Jeannot E, Lepreux S, Blanc JF, Blanché H, Le Bail B, Saric J, Laurent-Puig P, Balabaud C, Zucman-Rossi J: Clinical, morphologic, and molecular features defining so-called telangiectatic focal nodular hyperplasias of the liver. Gastroenterology 2005;128:1211-1218.

12 Paradis V, Benzekri A, Dargère D, Bièche I, Laurendeau I, Vilgrain V, Belghiti J, Vidaud M, Degott C, Bedossa P: Telangiectatic focal nodular hyperplasia: a variant of hepatocellular adenoma. Gastroenterology 2004;126: 1323-1329.

13 Sa Cunha A, Blanc JF, Lazaro E, Mellottee L, Le Bail B, Zucman-Rossi J, et al: Inflammatory syndrome with liver adenomatosis: the beneficial effects of surgical management. Gut 2007;5:307-309.

14 Laurent C, Trillaud H, Lepreux S, Balabaud C, Bioulac-Sage P: Association of adenoma and focal nodular hyperplasia: experience of a single French academic center. Comp Hepatol $2003 ; 23 ; 2: 6$.

15 Bioulac-Sage P, Laumonier H, Rullier A, Cubel G, Laurent C, Zucman-Rossi J, et al: Overexpression of glutamine synthetase in focal nodular hyperplasia: a novel easy diagnostic tool in surgical pathology. Liver Int 2009;29:459-465.
16 Van der Windt DJ, Kok NF, Hussain SM, Zondervan PE, Alwayn IP, de Man RA, et al: Case-orientated approach to the management of hepatocellular adenoma. Br J Surg 2006;93:1495-1502.

17 Toso C, Majno P, Andres A, Rubbia-Brandt L, Berney T, Buhler L, et al: Management of hepatocellular adenoma: solitary-uncomplicated, multiple and ruptured tumors. World J Gastroenterol 2005;11:5691-5695.

18 Cho SW, Marsh JW, Steel J, Holloway SE, Heckman JT, Ochoa ER, et al: Surgical management of hepatocellular adenoma: take it or leave it? Ann Surg Oncol 2008;15:27952803

19 Flejou JF, Barge J, Menu Y, Degott C, Bismuth $\mathrm{H}$, Potet $\mathrm{F}$, et al: Liver adenomatosis. An entity distinct from liver adenoma? Gastroenterology 1985;89:1132-1138.

20 Veteläinen R, Erdogan D, de Graaf W, ten Kate F, Jansen PL, Gouma DJ, et al: Liver adenomatosis: re-evaluation of aetiology and management. Liver Int 2008;28:499-508

21 Lepreux S, Laurent C, Blanc JF, Trillaud H, Le Bail B, Trouette H, Saric J, Zucman-Rossi J, Balabaud C, Bioulac-Sage P: The identification of small nodules in liver adenomatosis. J Hepatol 2003;39:77-85.

22 Bioulac-Sage P, Laumonier H, Rullier A, Cubel G, Laurent C, Zucman-Rossi J, et al: Overexpression of glutamine synthetase in focal nodular hyperplasia: a novel easy diagnostic tool in surgical pathology. Liver Int 2009;29:459-465.

23 Laumonier H, Bioulac-Sage P, Laurent C, Zucman-Rossi J, Balabaud C, Trillaud H: Hepatocellular adenomas: magnetic resonance imaging features as a function of molecular pathological classification. Hepatology 2008;48:808-818. 